\title{
Electro-Thermal Analysis of Oxide-Confined Vertical-Cavity Lasers
}

\author{
J. PIPREK ${ }^{1}$ ) \\ Electrical and Computer Engineering Department, University of California, \\ Santa Barbara, CA 93106, USA
}

(Received May 13, 2001; in revised form July 24, 2001; accepted August 29, 2001)

Subject classification: 07.05.Tp; 42.55.Px; 42.55.Sa; 68.35.Dv; 73.40.Kp; 85.60.Bt; S7.12

\begin{abstract}
The performance of oxide-confined vertical-cavity lasers (VCLs) can be severely limited by current leakage and self-heating. This paper investigates electrical and thermal effects of lateral oxidation on wafer-bonded $1.55 \mu \mathrm{m}$ VCLs. Measurements are combined with two-dimensional finite-element simulations to analyze internal device physics. Charged defects at the p-side fused interface are found to increase the threshold voltage as well as lateral current spreading. An optimum oxide aperture for minimum device heating is calculated. Loss reductions are predicted to lead to a minimization of self-heating with small apertures.
\end{abstract}

\section{Introduction}

In vertical-cavity surface-emitting lasers (VCLs), the optical cavity is formed by mirrors above and below the active region. The laser light propagates in vertical direction and typically exhibits a circular beam shape, ideal for coupling into optical fibers. Internally, the ligth passes the active layers in vertical direction, i.e., gain is provided over a short propagation distance only and the amplification per photon circulation is small. Therefore, the mirrors need to be highly reflective so that photons make many circulations before they are emitted. To achieve high reflectivity, distributed Bragg reflectors (DBRs) are used of two alternating layers with high refractive index contrast. With quarter-wavelength layer thickness, the reflected waves from all DBR interfaces add up constructively, allowing for DBR reflectivities above 99\% [1]. Dielectric DBRs like Si/SiO 2 provide a large refractive index contrast and a few layer pairs are sufficient for high mirror reflectivity [2]. However, semiconductor DBRs are often prefered to inject the current vertically through the mirror into the active region. Among semiconductor materials, AlAs/GaAs DBRs give a relatively high reflectivity, but the large band edge offset at the interfaces causes a high electrical resistance, especially in p-doped DBRs. Therefore, AlGaAs layers are frequently employed as well as sophisticated compositional grating and doping schemes at the interfaces [3]. The electric resistance and other heat sources may cause strong self-heating of VCLs so that good thermal conductivity is another essential requirement of VCL design.

The complexity and the interaction of electrical, thermal, and optical processes in VCLs often requires advanced computer simulation for device analysis and optimization. Using wafer-bonded $1.55 \mu \mathrm{m}$ VCLs as an example, this paper demonstrates how advanced simulations are used to design and analyze real devices. In order to gain realistic insight into VCL physics, the combination of simulations with measurements is especially emphazised. Section 2 describes the device structure. Section 3 outlines

\footnotetext{
1) Phone: (+805) 893 4051; Fax: (+805) 893 5440; e-mail: piprek@ece.ucsb.edu
} 
the numerical VCL model as well as the material parameters used to fit measurements. Sections 4 and 5 give details of the electrical and the thermal analysis, respectively.

\section{Long-Wavelength VCLs}

Long-wavelength VCLs $(1.3-1.6 \mu \mathrm{m})$ are currently of high interest for applications in fiberoptic communication systems. Compared to their in-plane counterparts, VCLs offer several advantages, including high fiber coupling efficiency, low power consumption, and low-cost wafer-scale fabrication and testing. In contrast to the rapid development of AlGaAs VCLs emitting at shorter wavelengths $(<1 \mu \mathrm{m})$, the performance of InP based long-wavelength devices is severely limited by disadvantageous material properties of the InGaAsP semiconductor system at long wavelengths. With the lower bandgap of the InGaAsP active region, Auger recombination enhances non-radiative carrier losses. With lower photon energy, free-carrier and intervalence band absorption (IVBA) lead to enhanced optical losses. Native InGaAsP/InP DBRs only allow a small variation of the refractive index that is about half the variation possible in AlGaAs. To obtain high DBR reflectances, a large number of mirror layers have to be grown causing significant diffraction losses [4]. InGaAsP also exhibits low thermal conductivity due to disorder scattering of phonons. Thus, thick InGaAsP/InP DBRs block the thermal flux to the stage and lead to a strong increase of the active region temperature in continuous-wave $(\mathrm{CW})$ operation.

Several advanced concepts of long-wavelength VCLs have been developed to overcome those limitations (for a review, see [5]). One of the most successful concepts is the utilization of InP/GaAs wafer fusion [6]. This way, traditional InP based active regions can be combined with superior GaAs based mirrors leading to record-high lasing temperatures [7, 8]. A wafer fused $1.55 \mu \mathrm{m} \mathrm{VCL}$ is shown schematically in Fig. 1 [9]. In this bottom-emitting device, 30 periods of $\mathrm{GaAs} / \mathrm{Al}_{0.67} \mathrm{Ga}_{0.33}$ As form the top DBR that is covered by a metal contact on a GaAs phase matching layer to enhance reflectivity. A ring contact can be used for top emission [10]. The mirror absorption is kept small by using relatively low p-doping (Table 1 ). The layer interfaces are parabolically graded to reduce interface electrical resistance [3]. Close to the fused interface, the mirror con-

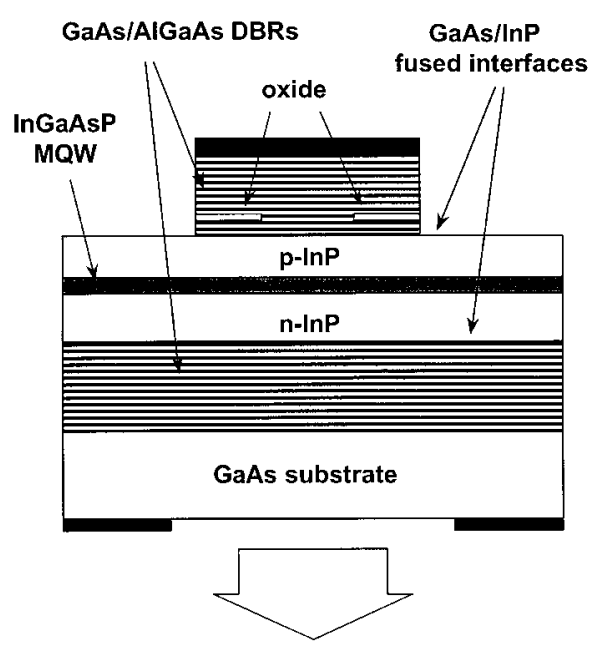
tains a $20 \mathrm{~nm}$ thin $\mathrm{Al}_{0.98} \mathrm{Ga}_{0.02} \mathrm{As}$ layer for lateral oxidation which is used for electrical and optical confinement. The InGaAsP multi quantum well (MQW) active region consists of 7 quantum wells with about $1 \%$ compressive strain and strain-compensating barriers. It is sandwiched between InP spacer layers which are about $300 \mathrm{~nm}$ thick to give a total

Fig. 1. Schematic structure of a bottom-emitting double-fused vertical-cavity laser with oxide confinement layer in the top mirror 
cavity thickness of three half wavelength. The bottom 28-period GaAs/AlAs DBR is pulse doped at all interfaces, in addition to uniform silicon doping. Binary DBR layers are used for high reflectivity and good thermal conductivity.

\section{Model and Parameters}

Based on the complexity of VCL physics, numerical simulations often need to combine electrical, thermal, optical, and gain calculations. Many previous publications on numerical VCL modeling focus on optical properties and neglect electrical or thermal aspects of VCL physics (e.g., [11, 12, 13]). Few papers on comprehensive numerical VCL simulations are published so far $[14,15,16]$, however, these papers do not analyze electrical and thermal effect of oxide confinement. In order to study those effects, a comprehensive two-dimensional VCL model is used here which has produced excellent agreement with a variety of measurements [15, 17, 18]. Carrier transport is simulated using a finite-element drift-diffusion model. The heat flux equation is included to address selfheating effects. Gain calculations are based on $4 \times 4 \mathbf{k} \cdot \mathbf{p}$ bandstructure computations for the strained quantum wells [19]. The transmission matrix method is employed for optical simulation [20] including temperature effects on layer thickness, absorption, and refractive index. The lateral optical modes are given by Bessel functions which are adjusted to measured near fields.

Such a comprehensive model includes various material parameters, some of which are not exactly known. An important first step of every device simulation is to identify critical parameters and to calibrate them using measured device characteristics. In our case, critical parameters are thermal and electrical conductivities of the DBR mirrors as well as the concentration of charged defects at the fused interface. Current-voltage and thermal measurements are employed to obtain realistic numbers (details are given below). Previous investigations have utilized measured variations of threshold current and slope efficiency with DBR diameter and with temperature to identify additional parameters [15]. Table 1 summarizes the results of this parameter calibration which leads to an excellent agreement between measurements and simulations.

Table 1

Room-temperature parameters of the double-fused VCL as used in the simulation ( $l$ layer thickness, $N_{\text {dop }}$ doping, $\mu_{z} / \mu_{r}$ majority carrier mobility (vertical/lateral), $n$ refractive index, $\alpha$ absorption coefficient, $\kappa_{z} / \kappa_{r}$ thermal conductivity (vertical/lateral)

\begin{tabular}{lllllll}
\hline $\begin{array}{l}\text { parameter } \\
\text { unit }\end{array}$ & $l$ & $\begin{array}{l}N_{\text {dop }} \\
1 / \mathrm{cm}^{3}\end{array}$ & $\begin{array}{l}\mu_{z} / \mu_{r} \\
\mathrm{~cm}^{2} / \mathrm{Vs}\end{array}$ & $n$ & $\begin{array}{l}\alpha \\
1 / \mathrm{cm}\end{array}$ & $\begin{array}{l}\kappa_{z} / \kappa_{r} \\
\mathrm{~W} / \mathrm{cm} \mathrm{K}\end{array}$ \\
\hline $\begin{array}{l}\text { p-Al }{ }_{0.67} \mathrm{Ga}_{0.33} \mathrm{As}(\mathrm{DBR}) \\
\text { p-GaAs (DBR) }\end{array}$ & 0.127 & $4 \times 10^{17}$ & see text & 3.05 & 11 & $0.10 / 0.12$ \\
p-InP (spacer) & 0.115 & $4 \times 10^{17}$ & see text & 3.38 & 11 & $0.10 / 0.12$ \\
p-InP (spacer) & 0.210 & $1 \times 10^{18}$ & 30 & 3.17 & 24 & 0.68 \\
$\mathrm{In}_{0.76} \mathrm{Ga}_{0.24} \mathrm{As}_{0.82} \mathrm{P}_{0.18}$ & 0.100 & $1 \times 10^{16}$ & 150 & 3.17 & 0.24 & 0.68 \\
$(\mathrm{QW})$ & 0.0055 & - & 100 & 3.6 & 54 & 0.043 \\
$\mathrm{In}_{0.48} \mathrm{Ga}_{0.52} \mathrm{Ass}_{0.82} \mathrm{P}_{0.18}$ & 0.008 & - & 100 & 3.4 & 54 & 0.043 \\
(barrier) & & & & & & \\
n-InP (spacer) & 0.310 & $5 \times 10^{18}$ & 4600 & 3.15 & 8 & 0.68 \\
n-GaAs (DBR) & 0.115 & $1 \times 10^{18}$ & $310 / 6200$ & 3.38 & 6 & $0.20 / 0.23$ \\
n-AlAs (DBR) & 0.134 & $1 \times 10^{18}$ & $310 / 6200$ & 2.89 & 3 & $0.20 / 0.23$ \\
n-GaAs (substrate) & 450 & $5 \times 10^{18}$ & 8000 & 3.38 & 5.8 & 0.44 \\
\hline
\end{tabular}



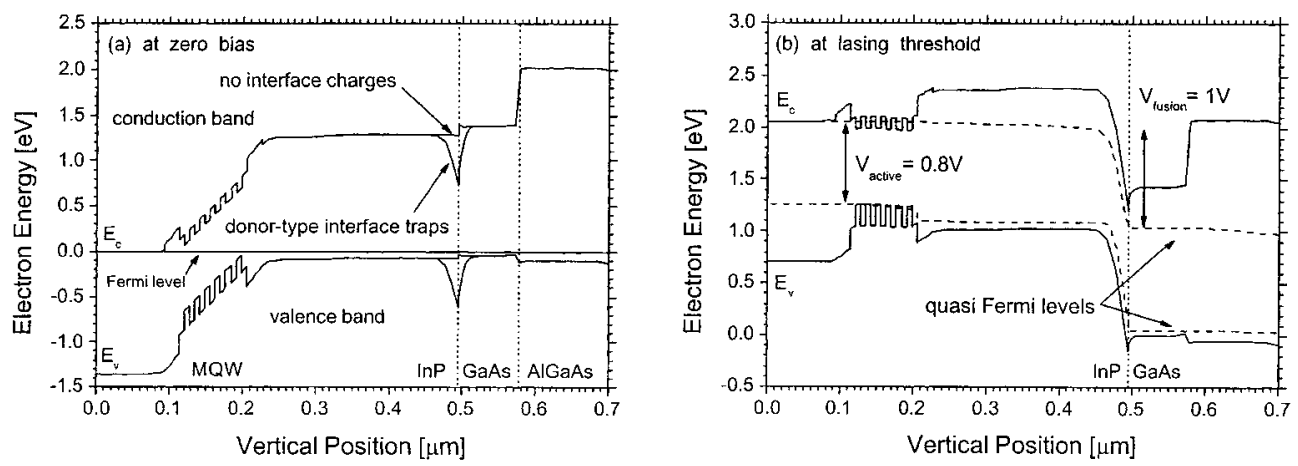

Fig. 2. Energy band diagram of active region and fused interface at the laser axis: a) zero bias, b) at threshold

\section{Electrical Analysis}

The carrier transport through the double fused VCL is affected by a large number of hetero-interfaces. One of the most critical interfaces is the p-side fused junction. Ideally, this interface between GaAs and InP exhibits only a small band offset [21]. However, in some fused VCLs, positively charged defects were found to create a large potential barrier in the valence band [22]. The nature of these donor-type defects is still unclear, however, high p-doping at the fused interface was found to reduce their influence on VCL performance [23]. Figure 2 shows the band diagram along the VCL axis, from the n-InP spacer layer, through MQW active region and fused interface, to the first AlGaAs layer of the top DBR. Without interface charges, only a small valence band offset of $0.05 \mathrm{eV}$ would slightly hinder hole injection. In comparison, a density of $10^{13} \mathrm{~cm}^{-2}$ mid-gap interface defects creates a potential barrier for holes that is more than $0.5 \mathrm{eV}$ high and that increases the VCL electric resistance. At lasing threshold, these interface defects lead to an additional voltage drop of about $1 \mathrm{~V}$ which increases the threshold voltage significantly (Fig. 2). Ideally, the threshold voltage should not be much larger than the QW Fermi level separation of about $0.8 \mathrm{~V}$ [24].

with interface defects

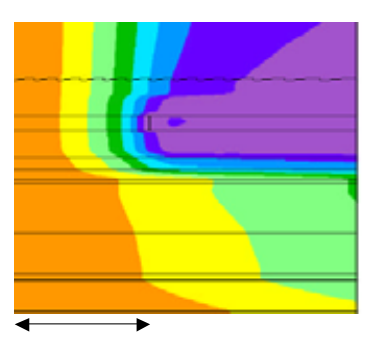

oxide aperture radius without interface defects

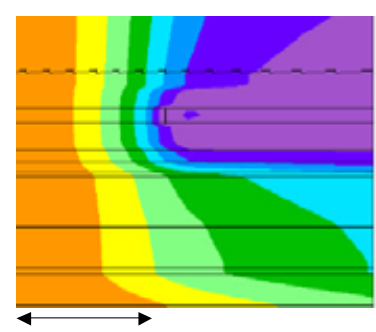

oxide aperture radius

Fig. 3. (colour) 2D current density contours $j(r, z)$ in the center part of the VCL with lateral oxide confinement layer: with (left) and without (right) positively charged defects at the fused interface (the left border is the laser axis, oxide aperture radius $=3.5 \mu \mathrm{m}$ ) 


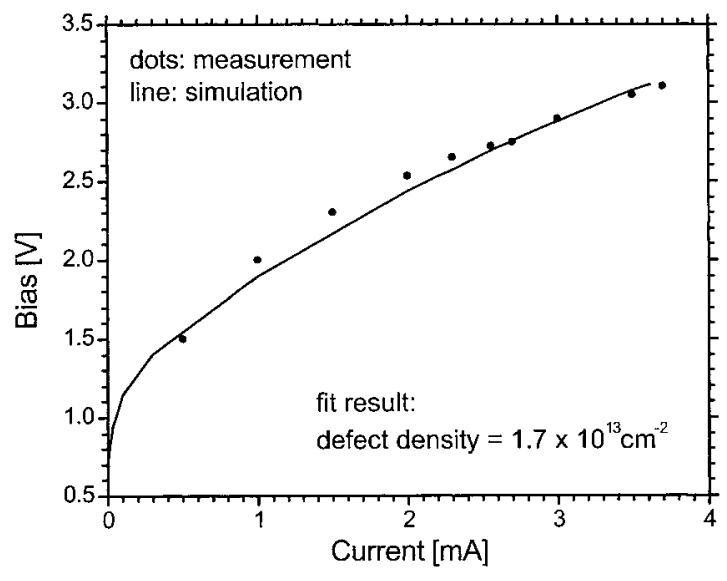

Fig. 4. Comparison of calculated (solid) and measured (dots [23]) current-voltage characteristics

A critical design issue of VCLs is the reduction of current leakage. In general, two leakage mechanisms can be distinguished near the active region: vertical and lateral leakage. Vertical leakage is caused by carriers that leave the active region in vertical direction by thermionic emission. The band diagram in Fig. 2 illustrates that thermionic emission of electrons into the lowly doped p-InP spacer is more likely than the leakage of holes in opposite direction. In our example device, vertical electron leakage is only relevant at high temperatures. It can be reduced when the MQW is sandwiched by InGaP stopper layers with slightly larger bandgap as shown in Fig. 2 [25].

Lateral leakage is mainly caused by hole spreading currents in p-doped layers above the active region as well as by ambipolar carrier diffusion within the quantum wells. Shrinking oxide confinement within the top DBR enhances lateral leakage currents. The spreading current is especially severe with positively charged defects at the p-side fused interface. After being funneled through the oxide aperture, holes encounter the high interface resistance which forces them to spread out laterally before entering the InP layer. This partially eleminates the confinement effect of the oxide layer. The current contour plot in Fig. $3^{2}$ ) illustrates the influence of charged interface defects. Each contour section represents $12.5 \%$ of the total current. Narrow contours indicate a high current density near the rim of the oxide aperture. Lateral current spreading between oxide and fused interface is much stronger with (left) than without (right) interface defects. Figure 4 shows the fit to the measured current-voltage curve, which results in a defect density of $1.7 \times 10^{13} \mathrm{~cm}^{-2}$ for this device [22].

\section{Thermal Analysis}

The electric resistance of interfaces and bulk layers as well as nonradiative recombination in the active layers are main heat sources in VCLs. Joule heating depends on the local current density. It is essential to know the magnitude and location of these heat sources to correctly calculate the internal VCL temperature distribution. In the following, aperture size effects on the VCL self-heating near threshold are evaluated using a simplified electro-thermal analysis.

At laser threshold, the total heat power is approximately given by the voltage drop across the device times the injection current $P_{\mathrm{th}}=V_{\mathrm{th}} I_{\mathrm{th}}$. The VCL threshold voltage $V_{\text {th }}$ includes the resistance of many DBR interfaces which is hard to calculate correctly. Instead, current-voltage measurements on DBR stacks [26] are evaluated to obtain average DBR mobilities. In vertical direction, a non-Ohmic function of the current den-

\footnotetext{
${ }^{2}$ ) Colour figure is published online (www.physica-status-solidi.com)
} 
sity $\mu_{z}(j)=0.12 \mathrm{~cm}^{2} / \mathrm{Vs} \times\left(j\left[\mathrm{kA} / \mathrm{cm}^{2}\right]\right)^{-0.6}$ is extracted for the p-DBR. In lateral direction, the p-DBR hole mobility $\mu_{r}=220 \mathrm{~cm}^{2} / \mathrm{Vs}$ is much larger and so is the electron mobility in the $\mathrm{n}$-DBR (Table 1). The resistance of the p-doped $\mathrm{InP} / \mathrm{GaAs}$ interface is approximated by $8 \times 10^{-4} \Omega \mathrm{cm}^{2}$ and the p-contact resistance is $2 \times 10^{-4} \Omega \mathrm{cm}^{2}$ [26].

The dissipation of heat power inside the device is often characterized by the thermal resistance $R_{\mathrm{th}}$. This parameter is usually determined as the average active region temperature rise $\Delta T_{\mathrm{a}}$ divided by the heat power. The thermal resistance of VCLs is typically on the order of $1000 \mathrm{~K} / \mathrm{W}$, one order of magnitude larger than in edge-emitting lasers [27]. This high number results from the smaller size of the heat source as well as from the low thermal conductivity $\kappa$ of DBRs. For binary materials, the small DBR layer thickness restricts the phonon mean free path and therefore reduces the thermal conductivity. For our AlAs/GaAs n-DBRs, the average thermal conductivity is measured to be about one half of the expected average bulk value [28]. The DBR's thermal conductivity is larger in lateral direction than in vertical direction and it is strongly reduced by AlGaAs alloy scattering of phonons [29]. Based on those experimental results, anisotropic thermal conductivities are used as given in Tab. 1. An average temperature dependence of $\kappa \propto T^{-1.375}$ is included [30], and a value of $\kappa=0.2 \mathrm{~W} / \mathrm{cmK}$ is assumed for the oxide layer.

Lateral oxidation allows very small active areas and very low threshold currents $I_{\text {th }}$. In oxidized $1.55 \mu \mathrm{m}$ VCLs, $I_{\text {th }}=0.8 \mathrm{~mA}$ was reported with an oxide aperture of $d_{\mathrm{ox}} \approx 2.4 \mu \mathrm{m}$ resulting in a threshold current density of $j_{\mathrm{th}} \approx 18 \mathrm{kA} / \mathrm{cm}^{2}$ [31]. This large current density is due to lateral carrier leakage and optical scattering losses that increase with smaller oxide aperture. The measured dependence $j_{\text {th }}\left(d_{\text {ox }}\right)$ is given in Fig. 5 (dots) [9]. Two cases are compared to study the thermal effects of shrinking oxide apertures: 1) $j_{\mathrm{th}}=1 \mathrm{kA} / \mathrm{cm}^{2}$ (dashed line) and 2) $j_{\mathrm{th}}\left(d_{\mathrm{ox}}\right)$ as measured. In the first case, voltage and total heat power are reduced monotonically with shrinking oxide aperture. In the second case, a minimum voltage of $3.5 \mathrm{~V}$ at $d_{\mathrm{ox}}=14 \mu \mathrm{m}$ is calculated in good agreement with measurements [32]. A minimum heat power of $5.2 \mathrm{~mW}$ is generated at $d_{\mathrm{ox}}=8 \mu \mathrm{m}$ due to the monotonous reduction of the total current. It results in a maximum plug-in efficiency at that aperture.

Downsizing the active area causes a dramatic increase of the thermal resistance (Fig. 5). This is attributed to the reduced initial escape area for the heat generated in

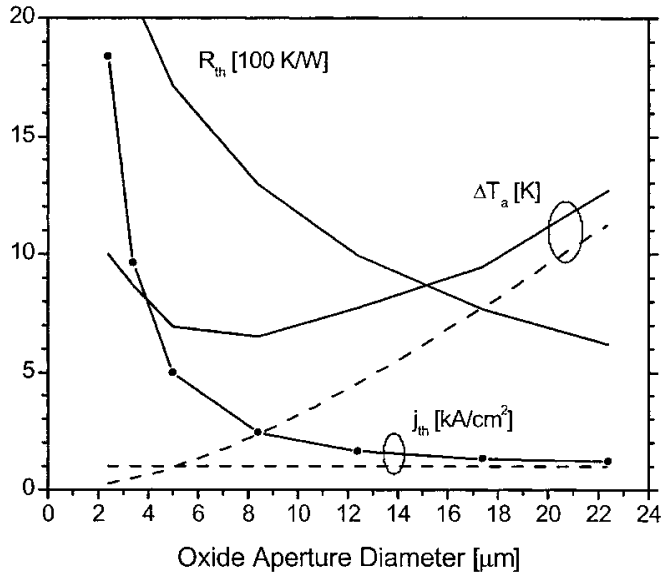
the center of the device. In our case, the simulations result in $R_{\mathrm{th}} \propto d_{\mathrm{ox}}^{-0.68}$.

The calculated average temperature rise $\Delta T_{\mathrm{a}}$ inside the active region of the oxidized VCL is plotted in Fig. 5. With constant threshold current density

Fig. 5. Threshold current density $j_{\text {th }}$ (dots: measured [9]), thermal resistance $R_{\mathrm{th}}$, and active region heating $\Delta T_{\mathrm{a}}$ as function of the oxide aperture diameter $d_{\text {ox }}$ (dashed: constant threshold current density) 
(dashed line), the self-heating at threshold would vanish for very small apertures. This is despite the strong increase of $R_{\text {th }}$ since the heat power is reduced even stronger $\left(I_{\mathrm{th}} \propto d_{\mathrm{ox}}^{2}\right)$. In the measured case of increasing threshold current density (solid line), a minimum temperature rise occurs at $d_{\mathrm{ox}}=8 \mu \mathrm{m}$ which is in good agreement with experimental results [31]. The dependence $j_{\mathrm{th}}\left(d_{\mathrm{ox}}\right)$ is dominated by increasing lateral leakage and optical losses with low apertures. Thus, by reducing such losses, the self-heating can be minimized.

\section{Summary}

In conlusion, the combination of advanced simulation with measurements allows for a detailed analysis of internal physical processes in VCLs. In wafer-fused devices, positively charged defects at the p-doped GaAs/InP interface can lead to a substantial increase of device voltage and spreading current. Thus, great care is required in the fusion process to diminish interface charges. Despite the increasing thermal resistance, self-heating can be lowered by lateral oxidation because of reduced heat power generation. An optimum oxide aperture of $8 \mu \mathrm{m}$ is found for our device that leads to minimum self-heating at threshold.

\section{References}

[1] T. E. SALE, Vertical Cavity Surface Emitting Lasers. Wiley, New York 1995.

[2] C. Wilmsen, H. Temkin, and L. A. Coldren (eds.), Physics of Strained Quantum Well Lasers, Cambridge University Press, Cambridge 1999.

[3] M. G. Peters, B. J. Thibeault, D. B. Young, A. C. Gossard, and L. A. Coldren, J. Vac. Sci. Technol. 12, 3075 (1994).

[4] D. I. Babić, Y. Chung, N. Dagli, and J. E. Bowers, IEEE J. Quantum Electron. 6, 1950 (1993).

[5] A. Karim, S. Bjorlin, J. Piprek, and J. Bowers, IEEE J. Quantum Electron. 6, 1244 (2000).

[6] A. Black, A. Hawkins, N. Margalit, D. Babic, J. Holmes, A. L., Y.-L. Chang, P. Abraham, J. Bowers, and E. Hu, IEEE J. Sel. Top. Quantum Electron. 3, 943 (1997).

[7] V. Jayaraman, T. Goodnough, T. Beam, F. Ahedo, and R. Maurice, IEEE Photonics Technology Letters 12, 1595 (2000).

[8] A. Karim, P. Abraham, D. Lofgreen, Y. Chiu, J. Piprek, and J. Bowers, Appl. Phys. Lett. 78, 2632 (2001).

[9] N. M. Margalit, J. Piprek, S. Zhang, D. I. Babić, K. Streubel, R. Mirin, J. R. Wesselmann, J. E. Bowers, and E. L. Hu, IEEE J. Sel. Top. Quantum Electron. 3, 359 (1997).

[10] N. Margalit, K. Black, Y. Chiu, E. Hegblom, K. Streubel, P. Abraham, M. Anzlowar, J. Bowers, and E. Hu, Electron. Lett. 38, 285 (1998).

[11] M. J. Noble, J. P. LoeHr, and J. A. LotT, IEEE J Quantum Electron. 34, 1890 (1998).

[12] D. Burak, J. Moloney, and R. Binder, IEEE J. Quantum Electron. 36, 956 (2000).

[13] G. P. Bava, P. Debernardi, and L. Fratta, Phys. Rev. A 63, 023816 (2001).

[14] G. Hadley, K. Lear, K. Warren, M. E. Choquette, J. Scott, and S. Corzine, IEEE J. Quantum Electron., 32, 607 (1996).

[15] J. Piprek, D. I. Babic, and J. E. Bowers, J. Appl. Phys. 81, 3382 (1997).

[16] O. Conradi, S. Helfert, and R. Pregla, IEEE J. Quantum Electron. 37, 928 (2001).

[17] J. Piprek, Y. A. Akulova, D. I. Babić, L. A. Coldren, and J. E. Bowers, Appl. Phys. Lett. 72, 1814 (1998).

[18] Y. M. Zhang, J. Piprek, N. Margalit, M. Anzlowar, and J. Bowers, IEEE J. Lightwave Technol. 17, 503 (1999).

[19] S. L. Chunan, Physics of Optoelectronic Devices, Wiley, New York 1995.

[20] L. A. Coldren and S. W. Corzine, Diode Lasers and Photonic Integrated Physics, Wiley, New York 1995.

[21] F. Salomonsson, K. Streubel, J. Bentell, M. Hammar, D. Keiper, R. Westphalen, J. Piprek, L. Sagalowicz, A. Roudra, and J. Behrend, J. Appl. Phys. 83, 768 (1998). 
[22] J. Piprek, A. Black, P. Abraham, E. L. Hu, and J. E. Bowers, in: Conf. on Lasers and ElectroOptics, Optical Society of America, Baltimore 1999 (pp. 458-459).

[23] A. K. Black, Wafer-fused vertical-cavity lasers, PhD thesis, University of California at Santa Barbara, 2000.

[24] M. Ortsiefer, R. Shau, G. Böhm, F. Köhler, and M.-C. Amann, Appl. Phys. Lett. 76, 2179 (2000).

[25] K. Black, P. Abraham, N. Margalit, E. Hegblom, Y.-J. Chiu, J. Piprek, J. Bowers, and E. Hu, Electron. Lett. 34, 1947 (1998).

[26] D. I. BABIĆ, Double-fused long-wavelength vertical-cavity lasers, $\mathrm{PhD}$ thesis, University of California at Santa Barbara, 1995.

[27] W. Both and J. PIPREK, J. Therm. Anal. 37, 61 (1991).

[28] J. Piprek, T. Troger, B. Schroter, J. Kolodzey, and C. S. Ih, IEEE Photonics Technol. Lett. 10, 81 (1998).

[29] G. Chen, C. L. Tien, X. Wu, and J. S. Smith, Trans. ASME, J. Heat Transf. 116, 325 (1994).

[30] S. AdachI, Physical Properties of III-V Semiconductor Compounds, Wiley, New York 1992.

[31] N. M. Margalit, D. I. Babić, K. Streubel, R. Mirin, R. L. Naone, J. E. Bowers, and E. L. Hu, Electron. Lett. 32, 1675 (1996).

[32] N. M. Margalit, High-temperature long-wavelength vertical-cavity lasers, $\mathrm{PhD}$ thesis, University of California at Santa Barbara, 1998. 